\title{
Bibliografía Literaria Hispanoamericana 1976
}

La investigación y la crítica de las letras hispanoamericanas continúan creciendo de prisa a pesar de los problemas que confrontan los editores: el ir de baja de los números de ejemplares que se publican, las dificultades en exportar libros causadas por las ordenanzas gubernamentales y la inflación espiral. Se vuelve cada día más esencial para quien desee saber lo que pasa en la literatura conocer los instrumentos, i.e., las bibliografías y las revistas culturales. En la última década, gracias a esfuerzos esmerados, aparecieron algunas bibliografías de primera categoría, en verdad indispensables en la investigación de un asunto a fondo. Algunas bibliografías sustanciales del teatro y de la novela demuestran que el interés continúa en la investigación de estos géneros. A mí me parece que las bibliografías, hasta las inferiores, sirven para iluminar, aunque con visibilidad intermitente, la acumulación de los materiales, facilitando de esta manera la tarea de separar lo significativo de lo ajeno al asunto, el grano de la barcia. La Revista Iberoamericana merece honor especial por haber publicado recientemente varias bibliografías de alta calidad.

Una bibliografía de naturaleza comprensiva, preparada especialmente para alumnos y bibliotecarios y corregida para la imprenta por Hensley C. Woodbridge y Dan Newberry, es Basic List of Latin American Materials in Spanish, Portuguese and French (Amherst, Mass.: SALALM Secretariat, University of Massachusetts Library at Amherst, 1975) (Seminar on the Acquisition of Latin American Library Materials, Bibliography, no. 2). Las secciones sobre las obras de consulta general, geografía, historia, lingüistica, filosofía, etc. fueron preparadas por varios especialistas. La materia en inglés no se incluye. Harvey L. Johnson compiló la quinta parte, "Literatura", 
pp. 101-150, que cubre las letras brasileñas e hispanoamericanas y se divide de la manera siguiente: relatos de la conquista y las civilizaciones indĭgenas; manuales y obras relacionadas con la cultura; estudios acerca de la novela y el cuento, el ensayo, el teatro, la poesïa, las literaturas nacionales, separadas éstas en las secciones de crítica y escritura creadora, y revistas. Se anotan las 327 entradas en la división "Literatura".

A Bibliography of Latin American Bibliographies, working paper no. A-2, compiled by Daniel Raposo Cordeiro and submitted for the 21st Seminar on the Acquisition of Latin American Library Materials (Bloomington, Indiana, Indiana University, May 2-6, 1976: SALALM Secretariat, University of Massachusetts Library at Amherst, Mass., 1976) es una compilación que cubre muchos campos. Los títulos de las obras de la literatura hispanoamericana recién publicadas figuran en las siguientes partes: general, biografias de individuos, disertaciones, indices de revistas, literatura y lengua; suplementa parcialmente Bibliography of Latin American Bibliographies de Arthur E. Gropp.

Latin America, Spain, and Portugal: An Annotated Bibliography of Paperback Books, $2^{\mathrm{a}}$. edición revisada (Washington, D.C.: Library of Congress, Hispanic Foundation, 1976) (Bibliographical Series, no. 14), fue recopilada por Georgette M. Dorn, quien con la ayuda de otros excluyó los títulos agotados y añadió los que acaban de publicarse. Contiene más de 2,200 títulos con anotaciones e incluye los títulos relativos a España y Portugal, además de los relacionados con la América Latina. Todos los campos de las humanidades y las ciencias sociales son incluidos; los de la literatura y la historia son los más numerosos.

Libros españoles ISBN 1976 (Madrid: International Standard Book Number, 1976) comprende en este suplemento toda la producción correspondiente a los ISBN asignados por la Agencia Española desde 1-11-1973 al 31-12-1975, es decir, un total de unos 40,000 tỉtulos editados o próximos a aparecer en el mercado en las fechas señaladas. El tomo se divide en las secciones siguientes: orden numérico de prefijos editoriales ISBN, lista alfabética de editores, indice de colecciones, indice de tîtulos, îndice de autores, îndice de materias. Las secciones 211-215 abarcan las literaturas española e hispanoamericana y los estudios crìticos; los títulos peninsulares predominan en todos los géneros. Es propósito de la Agencia Española, para un futuro próximo, incorporar a sus suplementos sucesivos la producción librera de los países hispanoamericanos que deseen ad- 
herirse al sistema ISBN a través de la misma.

David S. Zubatsky en "Annotated Bibliography of Latin American Author Bibliographies," Chasqui, 6(1), noviembre 1976, 43-64, intenta presentar una bibliografía anotada de bibliografías preparadas o por los autores mismos (novelistas, dramaturgos, poetas, ensayistas) o por otros que escriban sobre éstos. Las recopilaciones son tomadas de periódicos, revistas, disertaciones, tomos de homenaje, etc. La primera parte, referente a México, tiene 28 entradas en la sección de obras generales y 42 en la de escritores individuales. Las anotaciones son valiosas.

Dictionary of Contemporary Latin American Authors (Tempe, Arizona: Arizona State University, Center for Latin American Studies, 1975) fue compilada por David W. Foster con la colaboración de 25 especialistas en la literatura latinoamericana: comprende las entradas de unos 250 autores contemporáneos, excluyendo a los brasileños; la producción de cada autor es evaluada críticamente y se registran sus obras principales; se cubren todos los géneros, incluso el ensayo y la crìtica literaria. Horacio Jorge Becco y David William Foster en su comprensiva La nueva narrativa hispanoamericana, bibliografia (Buenos Aires: Casa Pardo, 1975) incorporan 2,257 entradas en una sección clasificada de estudios generales y una recopilación de obras por 15 destacados novelistas y escritores de cuentos y crĭtica de ellos.

El tomo II del Indice informativo de la novela hispanoamericana: Las Antillas (Río Piedras, P.R.: Universidad de Puerto Rico, Editorial Universitaria, 1974) de Edna Coll contiene observaciones propias sobre la novela hispanoamericana, reproduce las opiniones expresadas por otros y proporciona información bibliográfica acerca de los escritores discutidos. Los novelistas son todos de Puerto Rico, la República Dominicana y Cuba. Es un buen libro de consulta.

"Fuentes bibliográficas para el estudio de la novela chilena (1843-1960)," por Lucỉa Guerra-Cunningham, Revista Iberoamericana, 42 (96-97), julio-diciembre de 1976, 601-619, es ordenada en 5 categorias: literatura chilena en general; movimientos literarios y tendencias en particular; autores y novelas; espacios, temas y motivos en la novela chilena; material bibliográfico. Raymond L. Williams recopila 149 títulos de novelas colombianas, divididas por autor en orden alfabético en "La novela colombiana, 1960-1974, una bibliografïa," Chasqui, 5(3), 1976, 27-39.

Leon F. Lyday y George W. Woodyard ponen al día su bibliografía crítica del teatro durante 34 años con su excelente $A$ Bibliog- 
raphy of Latin American Theater Criticism, 1940-1974 (Austin: University of Texas, Institute of Latin American Studies, 1976), que incluye más de 2,300 entradas de artículos, libros, disertaciones y reseñas de libros; los libros y las reseñas están con contrarreferencias numéricas. Margaret S. Peden en "Emilio Carballido, curriculum operum," Texto Critico, no. 3, 1976, 94-112, recopila en orden cronológico 147 entradas con notas que tratan de las obras de este dramaturgo mexicano. Esta bibliografía pone al dǐa la que originalmente fue publicada en 1967 en Latin American Theatre Review. La Biblioteca Nacional del Uruguay publicó Florencio Sánchez, 1875-1975, centenario de su nacimiento: bibliografía (Montevideo, 1975); para conmemorar el nacimiento del dramaturgo hace cien años.

José Luis Gómez-Martǐnez, en la nueva revista Los Ensayistas, boletin informativo, incluye "El ensayo como género literario: (una bibliografía selecta del estudio de él en el mundo hispánico), 1(1), marzo 1976, 19-24 y 1(2), octubre 1976, 34-37, recopilación arreglada en orden alfabético y con entradas acerca del ensayo español e hispanoamericano que se mezclan indiscriminadamente. Las entradas no se anotan y un alto porcentaje de ellas se relacionan con las letras peninsulares. Afortunadamente, en el futuro, las entradas hispanoamericanas van a separarse de las españolas.

Digna de estar a la cabeza de la lista de compilaciones referentes a las revistas es la del perito en bibliografǐas Arthur E. Gropp, director jubilado de the Columbus Memorial Library, Unión Panamericana: Bibliography of Latin American Bibliographies Published in Periodicals, I (A-D) y II (E-Z), con indice (Metuchen, N.J.: Scarecrow Press, 1976). Unas 9,700 referencias se sacan de más de 1,000 periódicos publicados antes y durante 1965; las entradas se arreglan por autor bajo tema, subdivididas por païs. Un indice alfabético de los nombres de personas, corporaciones, burocracias gubernamentales, títulos de series y de periódicos, temas y nombres geográficos se proporcionan junto con una clave de los periódicos citados. Hay una sección que se reserva exclusivamente a la literatura (II, 712-728), pero entradas relativas a ella aparecen en otras secciones. Más de 4 años se necesitaron para hacer la obra.

"Bibliographic Indexes to Periodical Literature in the Romance Languages" de Ana M. R. Lloréns en The Modern Language Journal, 60 (1-2), January-February 1976, 23-30, da una descripción panorámica de los indices bibliográficos principales y más especializados de los periódicos que tratan de las lenguas y literaturas románicas. Las entradas españolas e hispanoamericanas se hallan en 
una sección, pero entradas pertinentes aparecen en cada parte del artǐculo.

Barbara Cox, del Centro Latinoamericano de UCLA, Los Angeles, California, con la colaboración de unos 15 miembros de SALALM (Seminar for the Acquisition of Latin American Library Materials) prepara indices de unas 300 revistas - muchas contienen artículos relacionados con asuntos culturales- seleccionadas para incluirse en el volumen de 1975 de Hispanic American Periodical Index, a publicarse en julio de 1977. Este es un proyecto de mérito. $H A P I$ se publicará anualmente como adjunto a Reference Series del Centro Latinoamericano.

Vernon A. Chamberlin e Ivan A. Schulman por "La Revista Ilustrada New York": History, Anthology, and Index of Literary Selections (Columbia, Mo.: University of Missouri Press, 1976), hacen accesible información acerca de uno de los periódicos en español que florecieron en los Estados Unidos durante las últimas décadas del siglo XIX y los primeros años del XX. El índice de selecciones literarias incluidas en la revista entre 1886 y 1898 y el indice de autores y sus contribuciones registran colaboradores tales como José Martí, Manuel Gutiérrez Nájera, Baldomero Sanín Cano, Rubén Dario, Ricardo Palma, Jorge Isaacs, et al., quienes prueban que dicha revista era un órgano auténticamente literario. La antología comprende artỉculos raros o no coleccionados y poemas, algunos escritos especialmente para la Revista. Los datos bibliográficos sobre esta revista poco conocida son muy útiles.

Dos tomos, que abarcan los años 1972 y 1973 , continúan la publicación de la Guia a las reseñas de libros de y sobre Hispanoamérica ( $A$ Guide to Reviews of Books from and About Hispanic America), preparada por Antonio Matos (Detroit: Blaine Ethridge Books, 1965- ). (Iniciada en la Universidad de Puerto Rico en 1965 en forma mimeografiada, pronto se suspendió el proyecto). Las reseñas de 350 periódicos se incluyen en un índice y se resumen en la lengua en que están escritas. Los asuntos que se discuten pertenecen a las ciencias sociales y las humanidades. Hay poca duplicación de materia en la Guia y Handbook of Latin American Studies.

Indices de suplementos culturales de Rio de la Plata (Montevideo: Libreria Internacional "El Gaucho," 1976) van a ser una serie de indices clasificados por autor y asunto de artículos en los suplementos culturales de La Prensa, La Nación, La Opinión, Clarín (publicados en Buenos Aires) y El Dia (publicado en Montevideo). El tomo que cubre el año de 1975 en El Día ya salió de la prensa. 
José Ortega en "Bibliografía selecta de la literatura boliviana (1969-1974)," Revista de Critica Literaria Latinoamericana, no. 1, 1975, 159-169, nos pone al corriente con su recopilación de las obras bolivianas (de creación la mayoría) que se clasifican por género y con anotaciones. El artículo contiene también una sección dedicada a la crítica.

Hernando Salazar Mejïa, Luis Dario y Gustavo Arbeláez en Bibliografía de autores antioqueños (desde 1822 hasta 1974), [Medellín]: Hernando Salazar M., 1975) preparada para el tercer centenario de la fundación de Medellín, registra los autores de esa ciudad y de sus obras editadas entre 1822 y 1974 . Las secciones de letras y asuntos referentes a la literatura son largas. Esta bibliografïa no tiene anotaciones, pero sí suministra un índice de los autores.

María Lya Niño de Rivas ofrece para el estudio de los escritores contemporáneos de su paîs una guía muy apreciada: "Escritores actuales de Venezuela: una bibliografía," Araisa, anuario 1975 (Caracas: Centro de Estudios Latinoamericanos "Rómulo Gallegos"), pp. 349-383. El mismo tomo contiene también "Rómulo Gallegos: bio-bibliografía," pp. 393-406, hacha por Raúl Díaz Legórburu. Araisa, iniciada con motivo de la celebración del primer aniversario de funcionamiento del Centro, exhibe alta calidad en sus artículos de crítica literaria, escritos de creación y reseñas; es un órgano digno de un centro cultural fundado en honor de Rómulo Gallegos y éste es de buen agüero para los anuarios futuros. Dicho Centro también publica Boletin Informativo que da noticias de sus diversas actividades $y$ varios departamentos.

Tres nuevas bibliografías pertinentes a la literatura venezolana se citan aquî: Lubio Cardozo, Bibliografia de bibliografías sobre la literatura venezolana en las bibliotecas de Madrid, Paris y Londres (Maracaibo: Universidad del Zulia y Universidad de Los Andes, 1975); Lubio Cardozo y Juan Pintó, Diccionario general de la literatura venezolana: autores (Mérida: Universidad de los Andes, Centro de Investigaciones Literarias, 1974) y Seudonimia literaria venezolana, con apéndice de José E. Machado (Mérida: Universidad de los Andes, Centro de Investigaciones Literarias, 1975) (Serie Bibliográfica, 6).

A la Dirección de Bibliotecas, Archivos y Museos se le debe el útil trabajo Referencias criticas sobre autores chilenos (Santiago de Chile: Ed. Biblioteca Nacional, 1975).

Hensley C. Woodbridge en Rubén Dario: A Selective Classified and Annotated Bibliography (Metuchen, N.J.: The Scarecrow Press, 1975) pone al corriente y amplïa dos bibliografías anteriores sobre el 
gran poeta nicaragüense, las que aparecieron en 1967 y 1968. Esta obra informativa, anotada con cuidado, presenta un análisis de las publicaciones referentes a Darío en categorías específicas junto con sugerencias para investigaciones futuras. Woodbridge merece nuestro agradecimiento por un trabajo bien hecho; examinó centenares de libros y artǐculos y hace referencia a unos 500. (Véase reseña de A. Roggiano en este mismo número)

El trabajo de James $H$. Ward, "Bibliografía de Luis Palés Matos," La Torre, 21 (79-80), enero-junio 1973, [i.e., junio 1975], 221-230, se divide en 3 partes: antologías de poesía, poemas y prosa. Las entradas revelan las enmiendas que el poeta hizo, corrigiendo y puliendo constantemente su obra. Su poesìa negra constituye sólo una porción pequeña de la producción poética.

Nicholas Hey en su "Bibliografía de y sobre Vicente Huidobro," Revista Iberoamericana, 41(91), abril-junio de 1975, 293-353, incluye muchos textos que nunca aparecieron en las colecciones de las obras del poeta, la mayor parte de ellos de naturaleza política y publicados en periódicos y revistas entre 1930-1940, y los primeros borradores de algunos de sus poemas. Hay 5 partes en esta bibliografía de las obras de Huidobro y 6 en la sección de escritos sobre él (libros, tesis, estudios de crítica, reseñas, etc.).

Rosa Rodrĭguez Reeves en "Bibliografïa de y sobre Manuel Rojas," Revista Iberoamericana, 42(95), abril-junio de 1976, 285-313, se divide en dos partes: (a) bibliografía de sus obras (novelas; traducciones de ellas; cuentos; colecciones de cuentos, ensayos y poesía; artículos y otras publicaciones); (b) lista de estudios sobre él (libros con informes acerca de él, artículos y materia inédita).

Hubert P. Weller divide su Bibliografia analitica y anotada de y sobre Martín Adán (Rafael de la Fuente Benavides) (1927-1974) (Lima: Instituto Nacional de Cultura, 1975) en 2 partes -la primera arreglada en orden cronológico y subdividido en 3 secciones: (a) clasificación de su poesía $\mathrm{y}$ prosa $\mathrm{y}$ estudios bibliográficos; (b) análisis bibliográfico en orden alfabético de la obra con fechas de publicación, referencias bibliográficas a todas las publicaciones integras y parciales e indicaciones respecto a las distintas versiones; (c) lista completa de las publicaciones de Adán, incluso las reediciones de sus obras- 16 entradas. La segunda parte consiste en una bibliografïa anotada sobre Adán (artïculos, ensayos, reseñas, referencias anecdóticas y biobibliográficas de menor importancia - 533 entradas). Esta es una bibliografía ejemplar. 
Martha Paley Francescato en "Selected Bibliography [of Julio Cortázar] (1938-1976)," Books Abroad, 50(3), Summer 1976, 513-516, da una lista de las obras de Cortázar, libros sobre Cortázar, traducciones al inglés de sus obras y artículos de crítica literaria en inglés sobre el autor. (Véase RI, Nos. 84-85) Bradley A. Shaw dio a luz Latin-American Literature in English Translation, An Annotated Bibliography (New York: New York University, 1975); abarca todos los géneros; los títulos se arreglan de acuerdo con la lengua y la cultura; hay una lista especial de obras de las islas del Caribe y de la Guayana Francesa.

Jorge J. Rodrĭguez-Florido, "Bibliografía de y sobre Ciro Alegrǐa," Chasqui, 4(3), mayo 1975, 23-54, se divide en novelas; cuentos, leyendas y relatos; colecciones de cuentos y novelas; colecciones de artículos periodísticos y libros de ensayos; fragmentos de novelas, cuentos y adaptaciones; traducciones de sus obras; ensayos, reseñas y artículos periodísticos; poesĩa y poemas en prosa; piezas teatrales; entrevistas e intervenciones orales; prólogos; y crítica sobre su obra -las entradas más numerosas de la bibliografía. Hay 336 entradas en toda la bibliografía.

Ignacio del Río ofrece un trabajo útil en su Guia del Archivo Franciscano de la Biblioteca Nacional de México (México: UNAM, Instituto de Investigaciones Bibliográficas, 1975). La Guia de las bibliotecas universitarias argentinas (Bahïa Blanca: Centro de Investigación Bibliotecológica de la Universidad Nacional del Sur, 1976) da información sobre publicaciones de 189 bibliotecas pertenecientes a 26 universidades argentinas: especialidad de la institución, material bibliográfico, sistema de catalogación y clasificación, etc.

El Centro de Estudios Iberoamericanos de la Universidad de California, San Diego, suministra Guide to Selected Ibero-American Holdings (recopilada por Judith Keene, Marguerite Norton, et al., 1975), que da breves descripciones del material investigado y colecciones especiales en su biblioteca; de las 11 secciones, las que se refieren a las letras hispánicas, mexicanas y Colecciones Especiales tienen más interés para nosotros. Gastón Somoshegyi-Szokol dio a luz la tercera de sus recopilaciones sobre las obras en la Biblioteca de la Universidad de California: Contemporary Chilean Literature in the University Library at Berkeley: A Bibliography and Introduction, Biographical Notes and Commentaries (Berkeley: University of California, 1975).

Claude L. Hulet recopila en "Dissertations in the Hispanic Languages and Literatures -1975," Hispania, 59(2), May 1976, 276-288, 
un poco menos de 100 disertaciones terminadas y más de 100 en preparación en la literatura y lengua hispanoamericanas. La adición de 45 departamentos de lingüistica de los cuales Hulet solicitó informes, da razón del aumento de títulos en el estudio de la lengua. Loretta E. Killinger en "American Doctoral Degrees Granted in Foreign Languages, 1974-1975," The Modern Language Journal, 60(1-2), January-February 1976, 58-62, incorpora los escritos sobre la literatura española e hispanoamericana, suplementando por lo tanto la lista de Hulet. Sobre Angel Flores, Bibliografia de escritores hispanoamericanos véase reseña de A. Roggiano en este mismo número.

University of Houston

Arizona State University
HARVEY L. JOHNSON DAVID W. FOSTER 
